\title{
Methotrexate-Related Pancytopenia: A Case Report
}

\author{
Yatin Mehta ${ }^{1}$ Chinmaya Nanda ${ }^{1}$ \\ ${ }^{1}$ Medanta Institute of Critical Care and Anesthesiology, Medanta \\ The Medicity, Gurgaon, Haryana, India
}

\begin{abstract}
Address for correspondence Chinmaya Nanda, MD, FIACTA, Medanta Institute of Critical Care and Anesthesiology, Medanta The Medicity, Sector 38, Gurgaon 122001, Haryana, India (e-mail: nandachinu@gmail.com).
\end{abstract}

\author{
Abstract \\ Keywords \\ - autoimmune disorders \\ - methotrexate \\ - pancytopenia
}

Methotrexate (MTX) is a commonly used drug in the treatment of autoimmune disorders. Although a very safe drug, it can rarely have serious side effects like pancytopenia and hepatotoxicity. We present here a case of methotrexate-induced pancytopenia leading to sepsis and multiorgan failure.

\section{Introduction}

Methotrexate (MTX) is a common drug used for the treatment of certain malignancies and autoimmune disorders including rheumatoid arthritis. It has been in widespread use because of its efficacy, tolerability, and relative lack of serious side effects. Most of the side effects of MTX include hepatic abnormalities and pneumonitis. Hematologic side effects like myelosuppression and pancytopenia are not uncommon side effects of MTX, but are underreported.

\section{Case Report}

A 51-year-old female with a diagnosed case of severe aortic regurgitation (AR) was admitted to our hospital from an outside hospital for heart failure. She had shortness of breath since last two months New York Heart Association (NYHA) Grade IV. She was a known case of Takayasu arteritis Type IIB and was on tablet Omnacortil $2.5 \mathrm{mg}$ once daily and tablet MTX $15 \mathrm{mg}$ once weekly since 2011.

On clinical examination, she was unable to lie down, had bilateral crepts on auscultation of chest, and her room air oxygen saturation was $93 \%$. Bilateral upper limb pulses were not palpable. Lower limb anterior and posterior tibial pulses were palpable. She had a history of allergic reaction to contrast dye during aortography performed two months ago in another hospital. Magnetic resonance angiography showed left internal carotid artery 95\% stenosed and complete occlusion of left vertebral artery.

Transthoracic echocardiography revealed thickened aortic valve with noncoaptation resulting in severe AR and no aortic stenosis. Aortic annulus was $2 \mathrm{~cm}$ and ascending aorta was $3.3 \mathrm{~cm}$ in diameter. She had mild mitral and tricuspid regurgitation, pulmonary artery systolic pressure of $33 \mathrm{~mm} \mathrm{Hg}$, global left ventricular hypokinesia with an ejection fraction of $45 \%$, normal right ventricular function, and increased left ventricular end diastolic pressure. Inferior vena cava was $8 \mathrm{~mm}$ in diameter and more than 50\% collapsing with respiration, no intracardiac clot, vegetation, or pericardial effusion. Her preoperative blood investigations showed hemoglobin of $10.4 \mathrm{~g}$, total leucocyte count of $8,400 / \mathrm{mm}^{3}$ with differential count within normal limits, platelet count of $1,47,000 / \mathrm{mm}^{3}$, and serum creatinine of 0.8 . Her preoperative blood, throat, and urine cultures were negative.

In view of her heart failure and nonresponse to conservative therapy, she was planned for aortic valve replacement (AVR). She had taken the last dose of MTX 5 days prior to the surgery. AVR was performed uneventfully and she was extubated the next day after 12 hours of overnight ventilation. The next two days of the postoperative course were uneventful. On the third postoperative day she had multiple episodes of bloody diarrhea. Her total leucocyte count (TLC) decreased to $300 / \mathrm{mm}^{3}$ and platelet count to $30,000 / \mathrm{mm}^{3}$. Peripheral blood smear showed no schistocytes. Urgent hematology and internal medicine consultation were taken. They opined the clinical picture as most likely MTX-induced pancytopenia and advised folinic acid $30 \mathrm{mg}$ intravenous 8 hourly and injection granulocyte colony stimulating factor G-CSF $300 \mu$ sc once daily. Her serum procalcitonin (PCT) level on the third postoperative day was $4.32 \mathrm{ng} / \mathrm{mL}$. On the fourth postoperative day her TLC decreased to $200 / \mathrm{mm}^{3}$ and platelet count was $40,000 / \mathrm{mm}^{3}$. She developed respiratory distress and hypotension for which she had to be intubated and mechanically ventilated. She was
DOI https://doi.org/

$10.1055 / \mathrm{s}-0039-3402326$

ISSN 2457-0206.
License terms

(ㅇ) (1) $\ominus \circledast$ 
started on broad spectrum antibiotics. Transthoracic echo performed on her revealed normal left ventricular function and normally functioning prosthetic valve at aortic position. Her blood pressure was low and she was on higher strengths of vasopressors to maintain her blood pressure. Her clinical condition continued to deteriorate. She received multiple units of packed red cells, fresh frozen plasma and platelet transfusions. She was hemodialysed due to falling urine output. On the eighth postoperative day her serum PCT level was $77.7 \mathrm{ng} / \mathrm{mL}$. All the blood, urine, and endotracheal secretion cultures showed no growth. She died on the ninth postoperative day due to multiorgan failure.

\section{Discussion}

MTX is one of the common disease-modifying anti-inflammatory drug used either as monotherapy or in combination with other drugs. MTX is a highly selective competitive inhibitor of the enzyme dihydrofolate reductase and hence reduces the production of thymidylate and DNA synthesis. MTX has wide acceptance because of good clinical response and less incidence of serious side effects. Most of the serious MTX toxicities that have been reported have focused on hepatic abnormalities and pneumonitis, while hematological reactions have been less emphasized. The incidence of MTX induced hematologic toxicities including leucopenia, thrombocytopenia, myelosuppression, and pancytopenia have been estimated to be $\sim 2$ to $4 \%$. Pancytopenia due to $\mathrm{MTX}^{1-3}$ is unpredictable and underreported. Pathogenesis of MTX induced pancytopenia is still unknown. Some of the known risk factors of MTX toxicity include impaired renal function, hypoalbuminemia, concurrent infection, advanced age, and coadministration of some drugs like NSAIDs, proton pump inhibitors and oral hypoglycemics. In most cases pancytopenia is transient and recovery occurs after discontinuation of MTX. MTX is excreted through kidneys; hence, MTX toxicity is common in patients with renal impairment. Folic acid supplementation has shown to reduce likelihood of liver impairment but protective effects on hematological toxicities have not been proven.

Many patients who are on MTX therapy have to undergo elective surgical procedures most commonly orthopedic in nature. There is no general consensus on how to manage disease modifying antirheumatic drugs (DMARD) in the perioperative setting. Many perioperative physicians stop MTX 2 weeks prior to elective surgery and restart it 2 weeks postoperatively. ${ }^{4,5}$ Most of the data regarding use of MTX in the perioperative setting are based on retrospective cohort studies. Most of the studies showed no difference in perioperative infection rates or wound complications between those who continued or discontinued MTX perioperatively.

Sany ${ }^{6}$ included 64 rheumatoid arthritis patients who underwent orthopaedic surgery in a randomized unblinded prospective 8-month follow-up study. A total of 32 patients continued MTX and 32 stopped it more than a week before surgery. All the study patients were on MTX mean dose of $10 \mathrm{mg} /$ week. No differences were found in relation to the occurrence of wound morbidity. No infections were registered in any group. In the study of carpenter, ${ }^{7}$ patients continued or stopped MTX according to orthopaedist or rheumatologist preferences, but no significant differences were found in patient demographic and disease features. However, there were more infections in the group who continued MTX perioperatively. Surprisingly, in a retrospective 1-year follow-up study by Murata ${ }^{8}$ there were fewer infections and rheumatoid arthritis flares in the MTX group.

Elective AVR has a very low incidence of postoperative mortality. According to STS ACSD Society of thoracic surgery Adult cardiac surgery database the mortality in isolated AVR is around 2 to $3 \%$. At our institution it was (2/127) $1.57 \%$ last year. This patient had severe pancytopenia leading to sepsis and septic shock refractory to appropriate therapy, probably due to MTX.9-14

\section{Conclusion}

MTX induced hematological toxicities are not uncommon and under reported. Perioperative physicians should be aware of these facts and patients should be closely monitored for associated risk factors especially impaired renal function. Patient on MTX therapy should be regularly monitored with complete blood count and liver function tests to identify myelosuppression and avoid the complications of pancytopenia. More studies are needed to frame guidelines regarding continuation of MTX in the perioperative setting. Maybe it should be stopped for a week or two before and after major surgery.

\section{Conflict of Interest}

None.

\section{References}

1 Gutierrez-Ureña S, Molina JF, García CO, Cuéllar ML, Espinoza LR. Pancytopenia secondary to methotrexate therapy in rheumatoid arthritis. Arthritis Rheum 1996;39(2):272-276

2 Howland WL. Methotrexate-associated bone marrow suppression following surgery. Arthritis Rheum 1988;31(12):1586-1587

3 Kuitunen T, Malmström J, Palva E, Pettersson T. Pancytopenia induced by low-dose methotrexate. A study of the cases reported to the Finnish Adverse Drug Reaction Register From 1991 to 1999. Scand J Rheumatol 2005;34(3):238-241

4 Bridges SLJ Jr, López-Méndez A, Han KH, Tracy IC, Alarcón GS. Should methotrexate be discontinued before elective orthopedic surgery in patients with rheumatoid arthritis? J Rheumatol 1991;18(7):984-988

5 Dias MJ. Re: Should methotrexate be stopped before surgery in patients with rheumatoid arthritis. J Hand Surg [Br] 2001;26(4):394

6 Sany J, Anaya JM, Canovas F, et al. Influence of methotrexate on the frequency of postoperative infectious complications in patients with rheumatoid arthritis. J Rheumatol 1993;20(7):1129-1132

7 Carpenter MT, West SG, Vogelgesang SA, Casey Jones DE. Postoperative joint infections in rheumatoid arthritis patients on methotrexate therapy. Orthopedics 1996;19(3):207-210

8 Murata K, Yasuda T, Ito H, Yoshida M, Shimizu M, Nakamura T. Lack of increase in postoperative complications with low-dose methotrexate therapy in patients with rheumatoid arthritis undergoing elective orthopedic surgery. Mod Rheumatol 2006;16(1):14-19 
9 Bridges SLJ Jr, Moreland LW. Perioperative use of methotrexate in patients with rheumatoid arthritis undergoing orthopedic surgery. Rheum Dis Clin North Am 1997;23(4):981-993

10 Grennan DM, Gray J, Loudon J, Fear S. Methotrexate and early postoperative complications in patients with rheumatoid arthritis undergoing elective orthopaedic surgery. Ann Rheum Dis 2001;60(3):214-217

11 Lim AY, Gaffney K, Scott DG. Methotrexate-induced pancytopenia: serious and under-reported? Our experience of 25 cases in 5 years. Rheumatology (Oxford) 2005;44(8):1051-1055

12 Lyssy KJ, Escalante A. Perioperative management of rheumatoid arthritis. Areas of concern for primary care physicians. Postgrad Med 1996;99(2):191-194, 199-201, 205-206
13 Perhala RS, Wilke WS, Clough JD, Segal AM. Local infectious complications following large joint replacement in rheumatoid arthritis patients treated with methotrexate versus those not treated with methotrexate. Arthritis Rheum 1991;34(2):146-152

14 Pieringer H, Stuby U, Biesenbach G. Patients with rheumatoid arthritis undergoing surgery: how should we deal with antirheumatic treatment? Semin Arthritis Rheum 2007;36(5):278-286

15 Sosin M, Handa S. Low dose methotrexate and bone marrow suppression. BMJ 2003;326(7383):266-267 